\title{
Effect of Electro-Acupuncture (EA) and Manual Acupuncture (MA) on Markers of Inflammation in Knee Osteoarthritis
}

This article was published in the following Dove Press journal: Journal of Pain Research

\author{
Guang-Xia Shi ${ }^{1}$ \\ Jian-Feng Tu $\mathbb{D D}^{2}$ \\ Tian-Qi Wang ${ }^{2}$ \\ jing-Wen Yang ${ }^{2}$ \\ Li-Qiong Wang ${ }^{2}$ \\ Lu-Lu Lin ${ }^{2}$ \\ Yu Wang' \\ Yong-Ting $\mathrm{Li}^{1}$ \\ Cun-Zhi Liu ${ }^{2}$
}

'Department of Acupuncture and Moxibustion, Beijing Hospital of Traditional Chinese Medicine Affiliated to Capital Medical University, Beijing, People's Republic of China; ${ }^{2}$ School of Acupuncture-Moxibustion and Tuina, Beijing University of Chinese Medicine, Beijing, People's Republic of China
Correspondence: Cun-Zhi Liu Email Icz_tg@I26.com
Background: Inflammation plays a significant role in the pathogenesis of knee osteoarthritis (KOA). Although both electro-acupuncture (EA) and manual acupuncture (MA) are known to influence systemic inflammation, little is known about the potential changes in inflammation as a working mechanism of EA and MA in KOA.

Methods: Data from the Acupuncture for Knee Osteoarthritis Trial (ATKOA) were used. Serum concentrations of inflammatory factors (tumor necrosis factor- $\alpha$ (TNF- $\alpha$ ), interleukin$1 \beta$ (IL-1 $\beta$ ), IL-6, IL-8, IL-18, IL-4, IL-10, IL-13, IL-15, IL-17, monocyte chemotactic protein-1 (MCP-1), CC-chemokine ligand 5 (CCL5), and cartilage degradation biomarkers (matrix metalloproteinase-1 MMP-1, MMP-3, MMP-13 and cartilage oligomeric matrix protein COMP)) were measured at baseline and after 8 weeks of treatment. Clinical outcomes were valid and reliable self-reported pain and function measures for osteoarthritis using the Western Ontario and McMaster Universities Osteoarthritis Index (WOMAC) and visual analogue scale (VAS) at baseline and post-treatment.

Results: Both 8-weeks EA and MA significantly reduced pro-inflammatory cytokines (TNF $\alpha$, IL-1 $\beta$ ), and cartilage degradation biomarkers (MMP-3, MMP-13) significantly increased the anti-inflammatory cytokine IL-13 compared with pre-treatment $(p<0.05)$. Further, the reduction of TNF- $\alpha$ was more significant in EA when compared to MA $(p=0.046)$. While there was no significant difference between groups in cytokines IL-1 $\beta$ $(\mathrm{p}=0.102)$, MMP-3 ( $\mathrm{p}=0.113)$, MMP-13 $(\mathrm{p}=0.623)$ or IL-13 $(\mathrm{p}=0.935)$. Moreover, in both EA and MA, the effect of acupuncture on the VAS and WOMAC function scale after 8 weeks is clinically important, although no significant differences were found between groups.

Conclusion: Eight weeks of both EA and MA seem to provide improvement in pain relief and function among individuals with mild to moderate knee OA. This benefit is partly mediated by changes of major inflammatory factors TNF- $\alpha$, IL-1 $\beta$ and IL-13.

Trial Registration: Controlled-Trials.com Identifier: NCT03274713.

Keywords: knee osteoarthritis, electro-acupuncture, manual acupuncture, inflammation

\section{Background}

Knee Osteoarthritis (KOA) is one of the most common diseases among older people and is a leading cause of disability. A number of risk factors are well recognized, including age, female sex, smoking, physical inactivity, and vitamin $\mathrm{D}$ and estrogen deficiency. ${ }^{1,2}$ In recent years, accumulating evidence indicates that inflammation has a critical role in KOA pathogenesis. ${ }^{3,4}$ Longitudinal studies demonstrate that higher systemic concentrations of pro-inflammatory cytokines predict increased radiographic progression of $\mathrm{KOA}$ as much as 5 years later. ${ }^{5}$ 
Besides direct effects on the joint, inflammatory mediators could affect muscle function and sensitize nerves leading to increased pain. ${ }^{6}$

Acupuncture treatment, which includes manual acupuncture (MA), electro-acupuncture (EA) and other types or other forms, has been used for a variety of pain conditions. ${ }^{7}$ It is also considered a potential non-pharmacologic intervention to prevent or to slow KOA progression although clinical guidelines have contradictory views. ${ }^{89}$ Our previous pilot study also showed that MA (three sessions per week for 8 weeks) is more feasible and safe compared with sham acupuncture for patients with KOA. ${ }^{10}$ Since acupuncture was known to reduce systemic concentrations of pro-inflammatory cytokines, the anti-inflammatory effect is suggested as a potential working mechanism. For example, EA at the ST36 acupuncture point reduced the lipopolysaccharide (LPS)-induced serum levels of cytokines TNF- $\alpha$, monocyte chemotactic protein-1 (MCP-1), and this anti-inflammatory mechanism mediated by the sciatic and vagus nerve. ${ }^{11}$ Inflammatory cytokine (IL-10) is crucial for analgesic and anti-inflammatory effects of MA in a model of inflammatory muscle pain. ${ }^{12}$ However, most of these studies use animal models, only a few studies evaluating the serum cytokine concentrations in KOA patients. The mechanisms responsible for acupuncture in KOA adults remain unknown. Therefore, the objective of the current study was to study the potential of cytokine modulation as a working mechanism of EA and MA for KOA patients using data from the Acupuncture for Knee Osteoarthritis Trial (ATKOA).

\section{Methods}

The current study used data from the Acupuncture for Knee Osteoarthritis Trial (ATKOA), which was had been registered in Clinical Trials. gov (ID: NCT03274713). In short, in this trial subjects willing to undergo testing and intervention procedures were included, using the following criteria: age from 45 to 75 , knee pain intensity $\geq 40$ on a 100 visual analogue scale (VAS) with a duration of more than 6 months, and radiographic evidence of OA (Kellgren grade II or III) based on weight-bearing radiographs. Eligible subjects were randomized to either an EA group that received electro-acupuncture or an MA group that received manual acupuncture.

Acupuncture treatment was semi-standardized: all participants underwent acupuncture needling at a selection of local or distant traditional acupuncture points have chosen by the acupuncturists according to the principles of traditional Chinese medicine. Needles were inserted at 6-7 local points, which included ST34 (Liangqiu), ST35 (Dubi), ST36 (Zusanli),
Heding, Neixiyan, GB33 (Xiyangguan), GB34 (Yanglingquan), SP9 (Yinlingquan), SP10 (Xuehai), LR7 (Xiguan), LR8 (Ququan) and ah shi points, and at 2-3 distal points, which included GB31 (Fengshi), GB36 (Waiqiu), GB39 (Xuanzhong), GB41 (Zulinqi), ST40 (Fenglong), ST41 (Jiexi), LR3 (Taichong), BL60 (Kunlun), SP6 (Sanyinjiao) and KI3 (Taixi). In the EA group, an electrical apparatus (HANS-200A acupoint nerve stimulator, Nanjing Jisheng Medical Co, Ltd.) producing a density wave with a frequency of $2 / 100 \mathrm{~Hz}$ was connected to the needles with alligator clips to stimulate pairs of needles inserted at ST36GB34 and ST34-SP10. The fixed current intensity was uniformly $0.2 \mathrm{~mA}$. In the MA group, needles were inserted at the same points, but no current was passed. The middle wire was cut, although the appearance of the unit was identical. Both EA and MA therapies consist of 24 sessions lasting $30 \mathrm{~min}$ each, administered over 8 weeks (usually three sessions per week).

\section{Randomization, Allocation Concealment and Blinding}

Eligible participants were randomly assigned to the EA group or MA group in a 1:1 ratio using a central web-based randomization tool. The blocked randomization sequence was generated with SAS 9.3 software (SAS Institute, Cary, NC, USA) by an independent statistician who was not involved in the implementation or statistical analysis of the trial. Randomization was stratified within the three enrolment hospitals using a random block size of 6 . The sequence was embedded into the software (Beijing Guide Technology Co, Ltd). All participants, as well as the statisticians who conducted the statistical analysis, were blinded to group allocation.

All participants gave informed consent prior to baseline testing and all procedures of ATKOA were in accordance with the Helsinki Declaration. The protocol was approved by the Research Ethical Committee of Beijing Hospital of Traditional Chinese Medicine Affiliated to Capital Medical University (2017BL-020-01).

\section{Demographics}

At baseline, age, gender, Education background, and Duration of disease were determined. The BMI was determined using measured body weight and height (without shoes).

\section{Inflammatory Cytokines}

A blood sample was taken at 8 a.m. on the baseline and the first day after 8 weeks. About $3 \mathrm{~mL}$ of blood was drawn into a procoagulant tube placed on ice and 
centrifuged at $2500 \mathrm{rpm}$ for $5 \mathrm{~min}$ at 9 a.m. ( $1 \mathrm{~h}$ later). The serum was divided into three portions, each about $0.5 \mathrm{~mL}$ and placed at $-80{ }^{\circ} \mathrm{C}$ for assay within 6 months. The cytokine assay was performed using Bio-Plex Pro multiassay technology (Bio-Rad Laboratories, Hercules, CA) which is based on the use of fluorescently dyed beads conjugated with monoclonal antibodies specific for eleven target cytokines (TNF- $\alpha$, IL-18, IL-1 $\beta$, IL-8, IL-13, IL-10, IL-4, IL-6, IL-15, IL-17, MCP-1). These beads were incubated with the sample, and then a secondary biotinylated antibody was added. Finally, the sample was placed in a dedicated flow cytometer with two lasers and associated optics to measure the different molecules bound to the surface of the beads and thus registers the cytokine concentrations. Human enzyme-linked immunosorbent assay (ELISA) kits (RayBiotech, Atlanta, GA, USA) were used for total MMP-1, MMP-3, MMP-13, and kits (R\&D Systems, Minneapolis, MN, USA) was used for the analysis of COMP and CCL5. Analyses are mixed by user. The optical density was measured at $450 \mathrm{~nm}$ using an automatic ELISA reader (Sunrise; Tecan, Mannedorf, Switzerland).

\section{Clinical Outcomes}

The response rate was calculated according to a change of $50 \%$ from baseline in Western Ontario and McMaster Universities Osteoarthritis Index (WOMAC) pain, stiffness and function scores at 8 weeks. Secondary outcomes were valid and reliable self-reported pain and function measures for osteoarthritis. Knee pain on the WOMAC pain subscale (0-20, higher scores indicating worse pain, $\mathrm{MCID} \geq 12 \%$ improvement from baseline) and VAS (0-100, higher scores indicating worse knee Pain; MCID 2.0 units) extrapolated from 20-mm MCID reported for $100-\mathrm{mm}$ visual analogue scales. ${ }^{13}$ Physical function was measured using the WOMAC function subscale (Likert version 3.1), scored from 0 to 68 with higher scores indicating worse function (minimal clinically important difference [MCID], 6 normalized units). ${ }^{14}$ Participants completed questionnaires at baseline and 8 weeks.

\section{Safety Evaluation}

Safety and tolerability of treatment were assessed at each visit. Safety was assessed by identifying adverse events (a treatment-related adverse event was any reported event first occurring or worsening in severity during treatment, compared to baseline period) using open-ended questions and a checklist including common acupuncture side effects. Adverse events were collected at each visit and up to 8 weeks after the end of treatment and were analyzed with regard to their seriousness, intensity, and causal relationship with treatment and outcome.

\section{Sample Size Calculation}

The exploratory nature of the study did not necessarily require a formal sample size calculation. Thus, a sample size of 60 participants (30/group) was determined to be sufficient to achieve the pragmatic purpose of the trial (ie, the collection of information, such as aggregate values of the outcomes and their variation, and feasibility-related information necessary for designing a future clinical trial) according to clinical experience.

\section{Statistical Analysis}

A value of $\mathrm{P}<0.05$ would be considered statistically significant. Measurement data were expressed by mean and standard deviation (mean $\pm \mathrm{SD}$ ), enumeration data expressed as a percentage. Mean between-group differences and two-sided $95 \% \mathrm{CI}$ are also presented to assess superiority. Paired Student's $t$-test was used to compare the difference in serum inflammatory biomarkers and cartilage degradation biomarkers pre-treatment and post-treatment in each group. The cytokine levels were non-normally distributed and consisted of non-detects. Concentrations below the lower limit of detection were considered as nondetectable and were left-censored. Hence, a standard nonparametric rank-based test (Mann-Whitney test) was applied without loss of information. All statistical analyses were performed using SPSS 13.0 for Windows (SPSS, Chicago, IL, USA).

\section{Results \\ Patient Characteristics}

A total of 60 subjects aged between 52 and 78 participated and had completed data. Two participants were repeatedly randomized and the remaining 58 were included in analysis, 28 in EA group and 30 in MA group. There were no significant differences in demographic factors (age, sex and BMI) between these participants and those excluded (data not shown). Overall, most of the patients (82\%) were women and the mean age was $58.89 \pm 6.75$ years, with a mean body mass index of $25.12 \pm 3.74$ and a mean disease duration of $69.93 \pm 56.69$ months. At baseline, serum levels of biomarkers were not significantly different between groups. The median level of TNF- $\alpha$ was $28.25 \pm 9.54 \mathrm{pg} / \mathrm{mL}$, of IL-1 $\beta$ was 1.43 $\pm 0.37 \mathrm{pg} / \mathrm{mL}$ in EA group, and TNF- $\alpha$ was $29.64 \pm 11.34 \mathrm{pg} /$ 
$\mathrm{mL}, \mathrm{IL}-1 \beta$ was $0.93 \pm 0.45 \mathrm{pg} / \mathrm{mL}$ in MA group. Moreover, WOMAC function $(2.14 \pm 1.46$ in EA, $2.57 \pm 1.48$ in MA, respectively) and the VAS scores $(54.82 \pm 11.90$ in EA, $54.77 \pm 8.38$ in MA, respectively) did not show significant differences between the two groups at baseline $(\mathrm{P}>0.05)$.

\section{Inflammatory Cytokines}

For patients in EA group, compared to pre-treatment the proinflammatory cytokines TNF- $\alpha(28.25 \pm 9.54 \mathrm{pg} / \mathrm{mL}$ VS 16.26 $\pm 4.97 \mathrm{pg} / \mathrm{mL}, \mathrm{p}=0.002), \mathrm{IL}-1 \beta(1.43 \pm 0.37 \mathrm{pg} / \mathrm{mL}$ VS 1.01 $\pm 0.65 \mathrm{pg} / \mathrm{mL}, \mathrm{p}=0.046), \mathrm{IL}-8(18.98 \pm 0.39 \mathrm{pg} / \mathrm{mL}$ VS 11.36 $\pm 0.31 \mathrm{pg} / \mathrm{mL}, \mathrm{p}=0.008), \mathrm{IL}-18 \quad(145.56 \pm 26.21 \mathrm{pg} / \mathrm{mL} \mathrm{VS}$ $90.27 \pm 26.78 \mathrm{pg} / \mathrm{mL}, \mathrm{p}=0.009)$, MCP-1 (127.64 $\pm 49.87 \mathrm{pg} /$ $\mathrm{mL}$ VS $73.45 \pm 49.90 \mathrm{pg} / \mathrm{mL}, \mathrm{p}=0.001$ ) (Figure 1), and cartilage degradation biomarkers MMP-3 $(2772.61 \pm 495.97 \mathrm{pg} /$ $\mathrm{mL}$ VS1279.00 $\pm 161.32 \mathrm{pg} / \mathrm{mL} \mathrm{P}=0.002)$ and MMP-13 (125.84 $\pm 37.74 \mathrm{pg} / \mathrm{mL}$ VS $70.74 \pm 25.68 \mathrm{pg} / \mathrm{mL}, \mathrm{p}=0.035$ ) were significantly lower post-treatment (Figure 2). The antiinflammatory cytokine IL-13 $(1.98 \pm 0.43 \mathrm{pg} / \mathrm{mL}$ VS 2.65 $\pm 0.24 \mathrm{pg} / \mathrm{mL}, \mathrm{p}=0.036$ ) were significantly higher posttreatment compared to pre-treatment. There were no significant increases or decreases in cytokine levels: CCL-5 $(2806.96 \pm 139.51 \mathrm{pg} / \mathrm{mL} \quad$ VS $2840.49 \pm 161.47 \mathrm{pg} / \mathrm{mL}$, $\mathrm{p}=0.073)$, COMP $(21.46 \pm 0.61 \mathrm{pg} / \mathrm{mL}$ VS21.40 $\pm 0.84 \mathrm{pg} / \mathrm{mL}$, $\mathrm{p}=0.720)$, MMP-1 $(30492.64 \pm 6921.87 \mathrm{pg} / \mathrm{mL}$ VS 30831.58 $\pm 6786.89 \mathrm{pg} / \mathrm{mL}, \mathrm{p}=0.861$ ) from pre- to post-treatment.

For patients in MA group, compared to pre-treatment the pro-inflammatory cytokines TNF- $\alpha(29.64 \pm 11.34 \mathrm{pg} / \mathrm{mL}$ VS $25.97 \pm 48.65 \mathrm{pg} / \mathrm{mL}, \mathrm{p}=0.043), \quad$ IL-1 $\beta \quad(0.93 \pm 0.45 \mathrm{pg} / \mathrm{mL}$ VS0.59 $\pm 0.33 \mathrm{pg} / \mathrm{mL}, \mathrm{p}=0.048$ ) (Figure 1), and cartilage degradation biomarkers MMP-3 (2665.93 $\pm 381.79 \mathrm{pg} / \mathrm{mL}$ VS $1330.68 \pm 63.20 \mathrm{pg} / \mathrm{mL}, \mathrm{p}=0.008)$ and MMP-13 $(124.06 \pm 47.49$ $\mathrm{pg} / \mathrm{mL}$ VS62.30 $\pm 26.52 \mathrm{pg} / \mathrm{mL}, \mathrm{p}=0.019$ ) (Figure 2) were significantly lower post-treatment. The anti-inflammatory cytokine IL-13 $(2.07 \pm 0.86 \mathrm{pg} / \mathrm{mL}$ VS $2.81 \pm 0.75 \mathrm{pg} / \mathrm{mL}, \mathrm{p}=0.024)$ were significantly higher post-treatment compared to pretreatment. There were no significant increases or decreases in cytokine levels: IL-8 $(19.95 \pm 0.44 \mathrm{pg} / \mathrm{mL}$ VS $19.26 \pm 0.36$ $\mathrm{pg} / \mathrm{mL}, \mathrm{p}=0.621), \mathrm{IL}-18 \quad(149.54 \pm 49.52 \mathrm{pg} / \mathrm{mL}$ VS 138.23 $\pm 48.65 \mathrm{pg} / \mathrm{mL}, \mathrm{p}=0.053)$, CCL-5 $(2806.96 \pm 139.51 \mathrm{pg} / \mathrm{mL}$ VS $2840.49 \pm 161.47 \mathrm{pg} / \mathrm{mL}, \mathrm{p}=0.073)$, COMP $(21.46 \pm 0.61$ $\mathrm{pg} / \mathrm{mL}$ VS $21.40 \pm 0.84 \mathrm{pg} / \mathrm{mL}, \mathrm{p}=0.720)$, MMP-1 (30492.64 $\pm 6921.87 \mathrm{pg} / \mathrm{mL}$ VS $30831.58 \pm 6786.89 \mathrm{pg} / \mathrm{mL}, \mathrm{p}=0.861)$, MCP-1 (128.56 $\pm 50.34 \mathrm{pg} / \mathrm{mL}$ VS $126.34 \pm 51.32 \mathrm{pg} / \mathrm{mL}$, $\mathrm{p}=0.546$ ) from pre- to post-treatment.

Both EA and MA have a significant reduction in proinflammatory cytokines (TNF- $\alpha$, IL- $1 \beta$ ) and cartilage degradation biomarkers (MMP-3, MMP-13) and a significant increase in anti-inflammatory cytokine IL13. Further, the reduction of TNFawere more significant in EA when compared to MA $(p=0.046)$. However, there was no significant difference between the EA and MA groups at 8 weeks in blood cytokine concentrations IL$1 \beta(p=0.102)$ MMP-3 ( $p=0.113)$, MMP-13 $(p=0.623)$ or IL-13 ( $\mathrm{p}=0.935)$.

The serum levels of inflammatory markers were generally low with $98.4 \%$ of IL-15, IL-17 and LIF undetectable in this sample, IL-6 in EA group and IL4, IL-10 in the MA group, so this was not further analyzed.
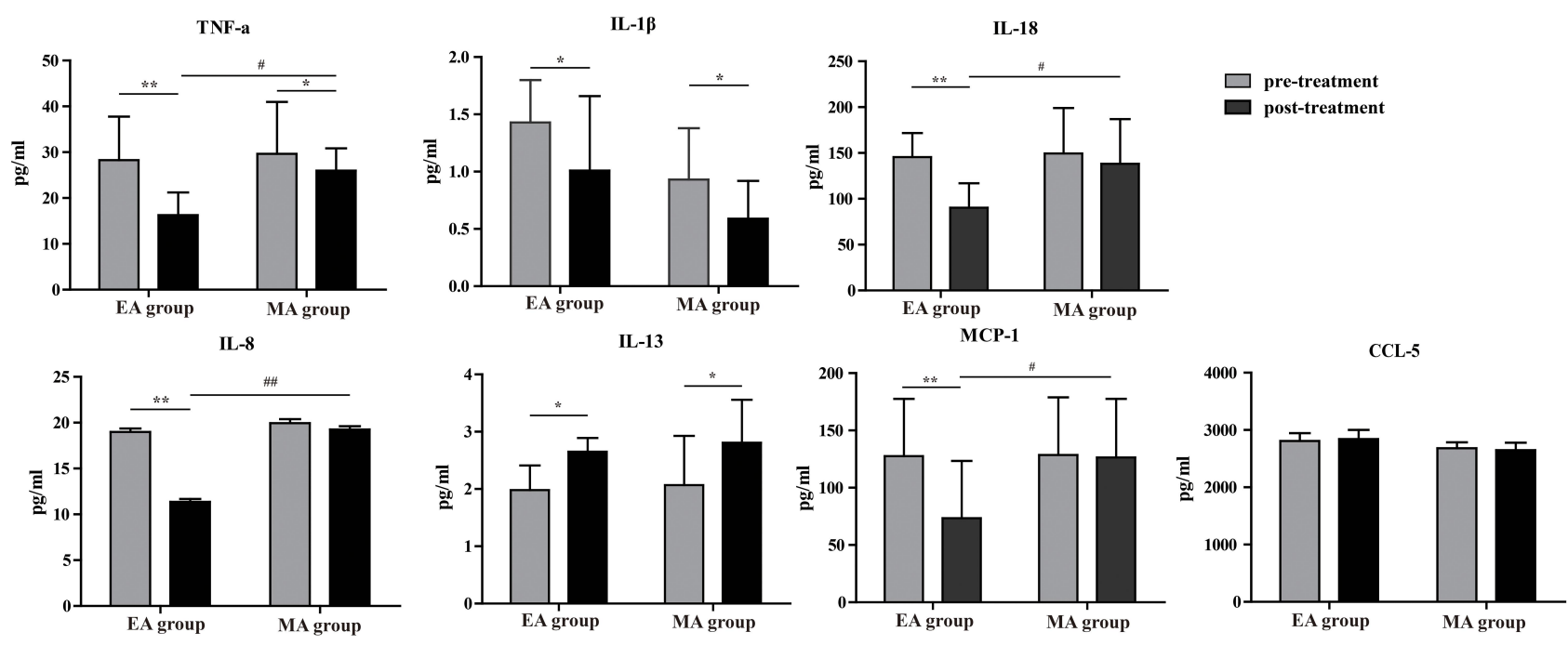

Figure I Comparison of plasma inflammatory cytokine concentrations. ${ }^{\#}$ Inter-group comparison: EA versus MA, ${ }^{\# p<0.05, ~}{ }^{\#} p<0.0$ I. ${ }^{* I n t r a-g r o u p ~ c o m p a r i s o n: ~ p r e ~ v e r s u s ~}$ post treatment, ${ }^{*} p<0.05$, $* * p<0.01$. 


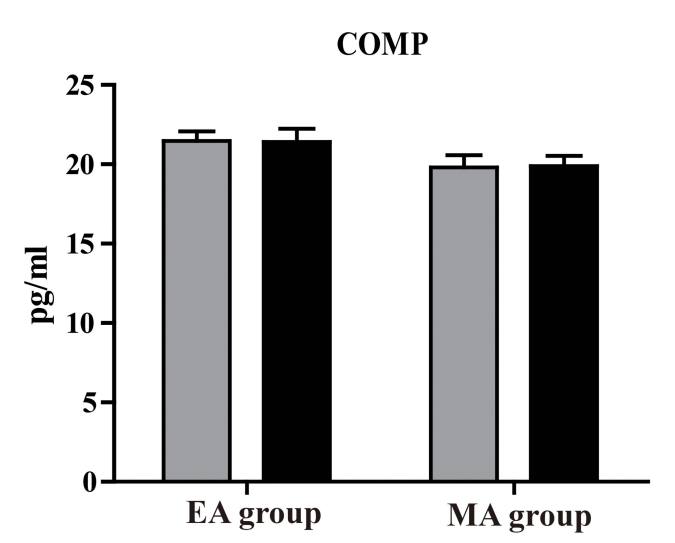

MMP-3

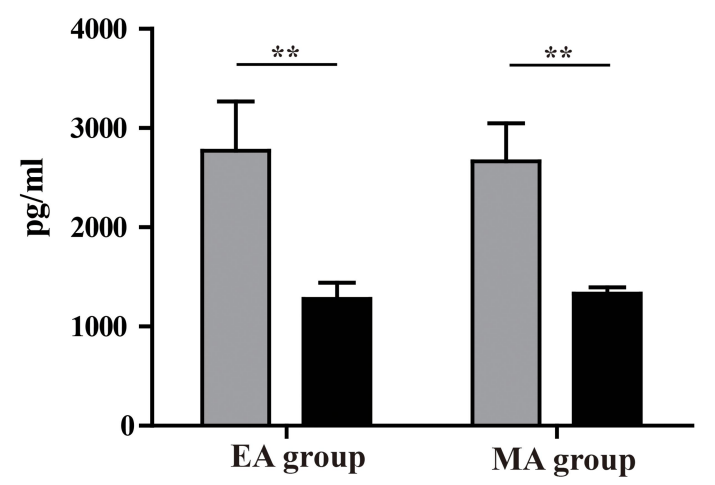

MMP-1

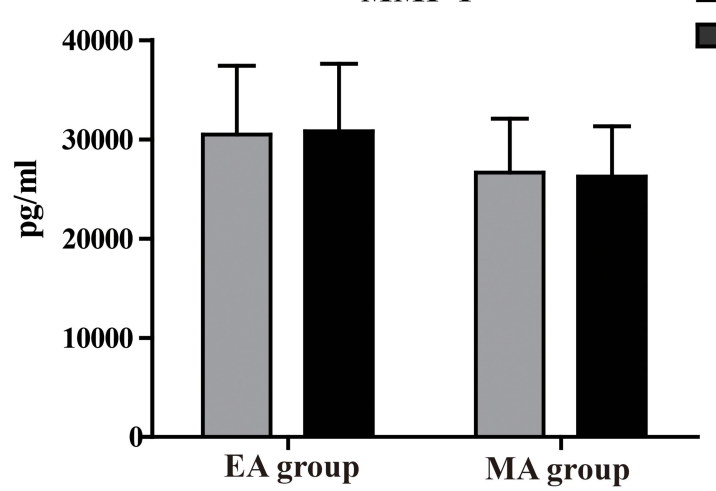

MMP- 13

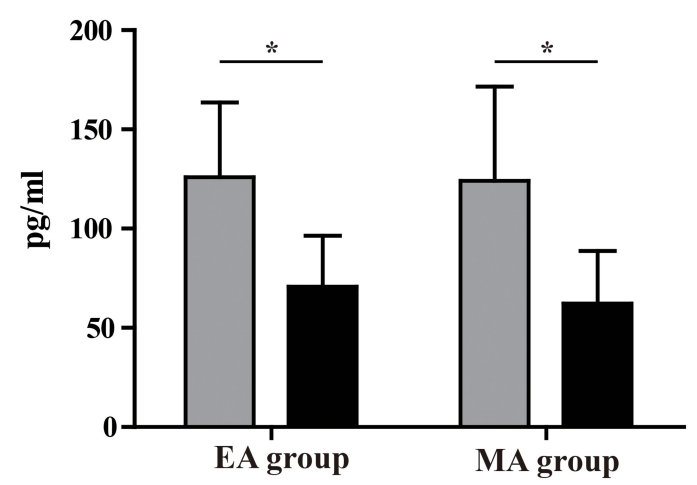

Figure 2 Comparison of plasma cartilage degradation biomarkers. *Intra-group comparison: pre versus post treatment, ${ }^{*} \mathrm{p}<0.05, * * \mathrm{p}<0.01$.

\section{Clinical Outcomes}

There were no clinically significant differences in the response rates in the EA group compared to the MA group at both 8 weeks ( $43 \%$ vs $30 \%, \mathrm{p}>0.05$, Table 1 ). Most secondary outcomes showed no difference. By week 8, the VAS pain score had decreased by 26.8 units in the EA group and 25.4 units in the MA group (Table 2). The mean change from baseline in WOMAC function was $9.5 \pm 3.5$ for EA and 8.3 \pm 3.2 for MA, although no significant differences were found between the two groups (Table 2).

\section{Safety}

No significant adverse effects were seen with respect to the procedure itself. A total of three adverse events were recorded: 11 in the EA group ( 2 needling pain after treatment, 9 hematoma) and 14 in the MA group (3 needling pain

Table I Response Rate of WOMAC Index in EA and MA Group

\begin{tabular}{|l|l|l|l|l|}
\hline \multirow{2}{*}{ Outcome } & \multicolumn{2}{|l|}{ Intention-to-Treat } \\
\cline { 2 - 5 } & $\begin{array}{l}\text { EA Group } \\
(\mathbf{n = 2 8})\end{array}$ & $\begin{array}{l}\text { MA Group } \\
(\mathbf{n = 3 0})\end{array}$ & P value & $\begin{array}{l}\text { Risk Difference } \\
\mathbf{( 9 5 \% ~ C l )}\end{array}$ \\
\hline Effective, $\mathrm{n}(\%)$ & $12(43)$ & $\begin{array}{l}9 \\
(30)\end{array}$ & 0.309 & 1.75 (0.59 to 5.16) \\
\hline Non-effective,n (\%) & $16(57)$ & $\begin{array}{l}21 \\
(70)\end{array}$ & & \\
\hline Between-group difference $(95 \% \mathrm{Cl})$ & $0.412(0.402$ to 0.42I) & \\
\hline
\end{tabular}

Abbreviations: EA group, electro-acupuncture group; MA group, manual acupuncture group. 
Table 2 Change in WOMAC Function and VAS Pain Scores in EA and MA Group

\begin{tabular}{|c|c|c|c|}
\hline Outcome & $\begin{array}{l}\text { EA Group } \\
(n=28)\end{array}$ & $\begin{array}{l}\text { MA Group } \\
(n=30)\end{array}$ & Between-Group Difference \\
\hline \multicolumn{4}{|c|}{ WOMAC function, mean $(95 \% \mathrm{Cl})$} \\
\hline Week 4 & $15.32(11.63$ to 19.01$)$ & 16.67 (I3.63 to 19.7I) & $-1.35(-6.17$ to 3.48$)$ \\
\hline Week 8 & II.39 (8.67 to I4.II) & 14.86 (II.97 to 17.74$)$ & $-3.46(-7.66$ to 0.73$)$ \\
\hline Week 12 & $10.04(7.42$ to 12.66$)$ & II.07 (8.59 to 13.55) & $-1.03(-4.85$ to 2.79$)$ \\
\hline Week 16 & 9.39 (6.46 to 12.26$)$ & $12.33(9.14$ to $15.3 \mid)$ & $-2.94(-7.30$ to 1.42$)$ \\
\hline \multicolumn{4}{|c|}{ VAS score $(0-100)$, mean $(95 \% \mathrm{Cl})$} \\
\hline Week 4 & 39.21 (24.44 to53.98) & 40.87 (24.79 to 56.95$)$ & $-1.66(-3.96$ to 0.64$)$ \\
\hline Week 8 & $26.04(12.20$ to 39.88$)$ & $31.00(|3.7|$ to $44.7 \mid)$ & $-4.96(-9.96$ to 0.04$)$ \\
\hline Week 12 & $21.83(6.19$ to 37.47$)$ & 25.83 (8.60 to 43.06$)$ & $-4.00(-8.47$ to 0.47$)$ \\
\hline Week 16 & $21.82(6.17$ to 37.46$)$ & 23.86 (3.86 to 43.86$)$ & $-2.04(-5.08$ to 1.00$)$ \\
\hline
\end{tabular}

Abbreviations: EA group, electro-acupuncture group; MA group, manual acupuncture group; WOMAC, Western Ontario and McMaster Universities Arthritis Index; VAS, visual analogue scale/score.

after treatment, 11 hematoma). These adverse events could remit spontaneously within 1 week. There were no serious adverse effects and all patients tolerated the treatments well.

\section{Discussion}

Both EA and MA have a significant reduction in proinflammatory cytokines (TNF- $\alpha, \mathrm{IL}-1 \beta$ ) and cartilage degradation biomarkers (MMP-3, MMP-13) and a significant increase in anti-inflammatory cytokine IL13. Further, the reduction of $\mathrm{TNF} \alpha$ was more significant in EA when compared to MA $(p=0.046)$. However, there was no significant difference between the EA and MA groups at 8 weeks in blood cytokine concentrations IL$1 \beta$, MMP-3, MMP-13 or IL-13.

Our research was based on the hypothesis that both EA and MA could act in an anti-inflammatory manner in individuals diagnosed with mild to moderate knee OA. We found that both 8 weeks EA and MA significantly reduced major pro-inflammatory cytokines (TNF- $\alpha$, IL$1 \beta$ ) and cartilage degradation biomarkers (MMP-3, MMP-13) and significantly increased the antiinflammatory cytokine IL-13 compared with pretreatment. Results in the EA group tended to be slightly better than those in the MA group that the reduction of TNF- $\alpha$ was more significant in EA when compared to MA. However, there was no significant difference between the EA and MA groups in blood cytokine IL-1 $\beta$, MMP-3, MMP-13 or IL-13.

A consensus report characterized decreases in individuals' pain intensity of approximately $10 \mathrm{~mm}$ was a clinically relevant pain improvement. By week 8 , the mean VAS pain score had decreased by 26.8 units in the EA group and 25.4 points in MA group (greater than MCID). Besides, changes of 6 points or more in WOMAC physical function were considered to be a clinical improvement. Improvement in WOMAC physical function at 8 weeks was of a clinically relevant in both EA ( 9.5 points $>$ pre-set MCID) and MA ( 8.3 points $>$ preset MCID). Thus, the results of this study demonstrated that both EA and MA had a clinically meaningful benefit in decreasing pain intensity and improving function, although there are no significant differences between groups due to the small sample size.

A number of underlying mechanisms have been proposed to mediate the analgesic and anti-inflammatory effects of acupuncture. Several studies show that both manual and electro-acupuncture produce analgesia through actions in the nervous system and the local tissues. ${ }^{7,15,16}$ These include opioids which reduce pro-inflammatory cytokines and norepinephrine. ${ }^{17}$ A study demonstrated a marked rise in plasma cortisol, which brought an expected anti-inflammatory effect in rats after EA treatment. ${ }^{18}$ The analgesic effect of EA and MA observed in the current study was also believed to be attributed to these previously reported mechanisms.

The development and progression of KOA are now believed to involve inflammation, even in the early stages of the disease. ${ }^{2}$ Among the pro-inflammatory cytokines involved in KOA, TNF- $\alpha$ and IL-1 $\beta$ are considered the major players; in particular, TNF- $\alpha$ with driving the inflammatory cascade, and IL- $1 \beta$ seems to be associated with cartilage destruction. These two cytokines, which are 
produced by chondrocytes, mononuclear cells, osteoblasts and synovial tissues, induce the production of a number of inflammatory and catabolic factors. ${ }^{3}$ In patients with KOA, levels of both TNF- $\alpha$ and IL-1 $\beta$ are elevated in the synovial fluid. TNF- $\alpha$ and IL- $1 \beta$ can act independently or in concert with other cytokines such as IL-6 and chemokines such as IL-8, MCP- 1 and CCl5. ${ }^{19-21}$ Besides, TNF- $\alpha$ and IL- $1 \beta$ could downregulate the synthesis of major extracellular matrix components (MMP-1, MMP-3 and MMP-13) by inhibiting anabolic activities of chondrocytes. ${ }^{22} \mathrm{~A}$ number of anti-inflammatory cytokines, such as IL-13, have been shown to be spontaneously elaborated by synovial membrane and cartilage, and are found in increased levels in the synovial fluid of OA patients. ${ }^{23}$ These cytokines exert their anti-inflammatory properties through a number of mechanisms, resulting in a decrease in the production of TNF- $\alpha$ and IL- $1 \beta$ and MMPs. MMP3 and MMP 13 is a major enzyme that targets cartilage for degradation. They not only target type II collagen in cartilage for degradation but also degrade proteoglycan, types IV and type IX collagen, osteonectin and perlecan in cartilage. ${ }^{24}$ This makes them prime targets for therapeutic strategies. Animal studies provide support for this approach, although only a few clinical studies have investigated the efficacy of blocking these inflammatory cytokines in the treatment of OA. Our result showed that 8 weeks of both EA and MA had an effect resulting in less knee pain and is partly mediated by changes in pro-inflammatory cytokines (TNF- $\alpha$, IL-1 $\beta$ ) and antiinflammatory cytokine IL-13, as well as cartilage degradation biomarkers (MMP-3, MMP-13) but not all inflammatory cytokines.

There could be several explanations for this apparent discrepancy. First, the levels of inflammatory cytokines were measured in serum, as opposed to synovial fluid, which did not allow us to detect local effects. IL-6 in serum and urine was not associated with clinical measures such as soft tissue swelling. ${ }^{25}$ Second, we included patients with mild to moderate knee osteoarthritis, some inflammatory markers may be undetectable in these people. Sakao et al reported that IL-6 expression was greater in patients with severe cartilage damage than those with mild cartilage damage. ${ }^{26}$ Furthermore, we saw no evidence of any statistical changes in COMP over the course of the 8-week intervention. It is possible that a longer intervention, beyond our 8 weeks could demonstrate attenuation of cartilage degradation or an increase information in the knee OA, but this would require further research. We measured total MMP-1 rather than active, and it is possible that active MMP-1 may have a closer relationship with knee structural measures, which also needs to be investigated in the future. ${ }^{27}$ Finally, given that knee structural changes do not explain all knee pain, the associations of inflammatory factors with structural changes may not translate to their associations with knee pain. Indeed, a few cross-sectional studies have reported inconsistent associations between inflammatory markers and knee pain, ${ }^{28}$ so this area remains controversial, and more studies are necessary to identify the roles of inflammation in knee pain.

Our result is in line with previous studies showing that transcutaneous electrical acupoint stimulation (TEAS) diminished the upregulation of pro-inflammatory factors in response to lower limb ischemia-reperfusion in patients undergoing limb ischemia-reperfusion. ${ }^{29}$ Moreover, in animal experimental studies it has been shown that acupuncture intervention has positive roles in relieving inflammatory reaction in rheumatoid arthritis by suppressing myocardial inflammatory cytokines (suppressing TNF- $\alpha$, IL-8, etc.), adjusting inflammatory reaction pathway. ${ }^{30}$

It is important to acknowledge the limitations of the present study. The interpretation of the data was limited by the small number of study participants. This may have influenced our ability to detect significant differences in all of the related inflammatory cytokines, and it is possible that with a larger sample size, more significant changes may be detected. Beyond this, while the focus of this study was limited to specific systemic biomarkers of inflammation and cartilage degradation (and each one of these biomarkers could be influenced by a number of systemic factors), it may be more appropriate to evaluate the effect of EA and MA more directly on the knee joint. While joint aspiration may be painful and invasive, the direct evaluation of biomarkers for inflammation and cartilage degradation from knee joint synovial fluid could allow the effects of acupuncture on knee joint health to be more accurately evaluated. The absence of a placebo is a serious limitation in this study, and we have tried to overcome this limitation by incorporating a sham treatment group in the future.

\section{Conclusion}

After 8 weeks of treatment, pain and joint function are improved in both MA and EA for patients with osteoarthritis of the knee. Besides, this benefit is partly mediated by changes in major inflammatory factors TNF- $\alpha$, IL- $1 \beta$, IL-13 and cartilage degradation biomarkers MMP-3, MMP-13. We understand that this study focuses only on the short-term changes in the clinical and markers. 
However, it will form a stepping stone for studies looking into the long term effects of EA and MA.

\section{Data Sharing Statement}

All the processed data were included in the current study. If reviewers or readers have any questions regarding our published data, they can contact the corresponding author [e-mail, lcz623780@126.com] for access to the original data.

\section{Ethics Approval and Consent to Participate}

The protocol was approved by the Research Ethical Committee of Beijing Hospital of Traditional Chinese Medicine Affiliated to Capital Medical University (2017BL-020-01).

\section{Acknowledgments}

We thank all the researchers and the subjects.

\section{Author Contributions}

All authors made substantial contributions to conception and design, acquisition of data, or analysis and interpretation of data; took part in drafting the article or revising it critically for important intellectual content; gave final approval of the version to be published; and agree to be accountable for all aspects of the work.

\section{Funding}

This study was supported by The 4th Project of China Association of Science and Technology Youth Talents Enrollment Project (2018QNRC001).

\section{Disclosure}

The authors declare that they have no competing interests.

\section{References}

1. Bijlsma JW, Berenbaum F, Lafeber FP. Osteoarthritis: an update with relevance for clinical practice. Lancet. 2011;377(9783):2115-2126. doi:10.1016/S0140-6736(11)60243-2

2. Felson DT. Osteoarthritis of the knee. $N$ Engl J Med. 2006;354 (8):841-848. doi:10.1056/NEJMcp051726

3. Fernandes JC, Martel-Pelletier J, Pelletier JP. The role of cytokines in osteoarthritis pathophysiology. Biorheology. 2002;39(1-2):237-246.

4. Berenbaum F. Osteoarthritis as an inflammatory disease (osteoarthritis is not osteoarthrosis!). Osteoarthritis Cartilage. 2013;21(1):16-21. doi:10.1016/j.joca.2012.11.012

5. Stannus OP, Jones G, Blizzard L, Cicuttini FM, Ding C. Associations between serum levels of inflammatory markers and change in knee pain over 5 years in older adults: a prospective cohort study. Ann Rheum Dis. 2013;72(4):535-540. doi:10.1136/annrheumdis-2011201047
6. Robinson WH, Lepus CM, Wang Q, et al. Low-grade inflammation as a key mediator of the pathogenesis of osteoarthritis. Nat Rev Rheumatol. 2016;12(10):580-592. doi:10.1038/nrrheum.2016.136

7. Han JS, Ho YS. Global trends and performances of acupuncture research. Neurosci Biobehav Rev. 2011;35(3):680-687. doi:10.1016/ j.neubiorev.2010.08.006

8. Witt C, Brinkhaus B, Jena S, et al. Acupuncture in patients with osteoarthritis of the knee: a randomised trial. Lancet. 2005;366 (9480):136-143. doi:10.1016/S0140-6736(05)66871-7

9. Corbett MS, Rice SJ, Madurasinghe V, et al. Acupuncture and other physical treatments for the relief of pain due to osteoarthritis of the knee: network meta-analysis. Osteoarthritis Cartilage. 2013;21 (9):1290-1298. doi:10.1016/j.joca.2013.05.007

10. Lin LL, Li YT, Tu JF, et al. Effectiveness and feasibility of acupuncture for knee osteoarthritis: a pilot randomized controlled trial. Clin Rehabil. 2018;32(12):1666-1675. doi:10.1177/0269215518790632

11. Torres-Rosas R, Yehia G, Peña G, et al. Dopamine mediates vagal modulation of the immune system by electroacupuncture. Nat Med. 2014;20(3):291-295. doi:10.1038/nm.3479

12. da Silva MD, Bobinski F, Sato KL, Kolker SJ, Sluka KA, Santos AR. IL-10 cytokine released from M2 macrophages is crucial for analgesic and anti-inflammatory effects of acupuncture in a model of inflammatory muscle pain. Mol Neurobiol. 2015;51(1):19-31. doi:10.1007/s12035-014-8790-x

13. Dworkin RH, Turk DC, McDermott MP, et al. Interpreting the clinical importance of treatment outcomes in chronic pain clinical trials: IMMPACT recommendations. $J$ Pain. 2008;9(2):105-121. doi:10.1016/j.jpain.2007.09.005

14. Tubach F, Ravaud P, Baron G, et al. Evaluation of clinically relevant changes in patient reported outcomes in knee and hip osteoarthritis: the minimal clinically important improvement. Ann Rheum Dis. 2005;64(1):29-33. doi:10.1136/ard.2004.022905

15. Zhao ZQ. Neural mechanism underlying acupuncture analgesia. Prog Neurobiol. 2008;85(4):355-375. doi:10.1016/j. pneurobio.2008.05.004

16. Luo F, Wang JY. Modulation of central nociceptive coding by acupoint stimulation. Neurochem Res. 2008;33:1950-1955. doi:10.1007/ s11064-008-9692-y

17. Ahsin S, Saleem S, Bhatti AM, Iles RK, Aslam M. Clinical and endocrinological changes after electro-acupuncture treatment in patients with osteoarthritis of the knee. Pain. 2009;147(1-3):60-66. doi:10.1016/j.pain.2009.08.004

18. Zhang RX, Lao L, Wang X, et al. Electroacupuncture attenuates inflammation in a rat model. J Altern Complement Med. 2005;11 (1):135-142. doi:10.1089/acm.2005.11.135

19. Lotz M, Terkeltaub R, Villiger PM. Cartilage and joint inflammation. Regulation of IL-8 expression by human articular chondrocytes. J Immunol. 1992;148(2):466-473.

20. Villiger PM, Terkeltaub R, Lotz M. Monocyte chemoattractant protein-1 (MCP-1) expression in human articular cartilage. Induction by peptide regulatory factors and differential effects of dexamethasone and retinoic acid. $J$ Clin Invest. 1992;90 (2):488-496. doi:10.1172/JCI115885

21. Alaaeddine $\mathrm{N}$, Olee $\mathrm{T}$, Hashimoto $\mathrm{S}$, Creighton-Achermann L, Lotz M. Production of the chemokine RANTES by articular chondrocytes and role in cartilage degradation. Arthritis Rheum. 2001;44 (7):1633-1643. doi:10.1002/1529-0131(200107)44:7<1633::AIDART286>3.0.CO;2-Z

22. Corciulo C, Lendhey M, Wilder T, et al. Endogenous adenosine maintains cartilage homeostasis and exogenous adenosine inhibits osteoarthritis progression. Nat Commun. 2017;8:15019. doi:10.1038/ ncomms 15019

23. Scott JL, Gabrielides C, Davidson RK, et al. Superoxide dismutase downregulation in osteoarthritis progression and end-stage disease. Ann Rheum Dis. 2010;69(8):1502-1510. doi:10.1136/ $\operatorname{ard} .2009 .119966$ 
24. Reboul P, Pelletier JP, Tardif G, Cloutier JM, Martel-Pelletier J. The new collagenase, collagenase-3, is expressed and synthesized by human chondrocytes but not by synoviocytes. A role in osteoarthritis. J Clin Invest. 1996;1; 97(9):2011-2019. doi:10.1172/JCI118636

25. Wang M, Sampson ER, Jin H, et al. MMP13 is a critical target gene during the progression of osteoarthritis. Arthritis Res Ther. 2013;15 (1):R5. doi:10.1186/ar4133

26. Sakao K, Takahashi KA, Mazda O, et al. Enhanced expression of interleukin-6, matrix metalloproteinase-13, and receptor activator of $\mathrm{NF}-\kappa \mathrm{B}$ ligand in cells derived from osteoarthritic subchondral bone. J Orthop Sci. 2008;13(3):202-210. doi:10.1007/s00776-008-1227-5

27. Otterness IG, Weiner E, Swindell AC, Zimmerer RO, Ionescu M, Poole AR. An analysis of 14 molecular markers for monitoring osteoarthritis. Relationship of the markers to clinical end-points. Osteoarthritis Cartilage. 2001;9(3):224-231. doi:10.1053/ joca. 2000.0379
28. Hannan MT, Felson DT, Pincus T. Analysis of the discordance between radiographic changes and knee pain in osteoarthritis of the knee. J Rheumatol. 2000;27(6):1513-1517.

29. Mo Y, Chen S, Yang L, et al. The effect of transcutaneous electrical acupoint stimulation on inflammatory response in patients undergoing limb ischemia-reperfusion. Mediators Inflamm. 2017;2017:8369737. doi:10.1155/2017/8369737

30. Xu Y, Hong S, Zhao X, et al. Acupuncture alleviates rheumatoid arthritis by immune-network modulation. Am J Chin Med. 2018;46 (5):997-1019. doi:10.1142/S0192415X18500520

\section{Publish your work in this journal}

The Journal of Pain Research is an international, peer reviewed, open access, online journal that welcomes laboratory and clinical findings in the fields of pain research and the prevention and management of pain. Original research, reviews, symposium reports, hypothesis formation and commentaries are all considered for publication. The manuscript

Submit your manuscript here: https://www.dovepress.com/journal-of-pain-research-journa management system is completely online and includes a very quick and fair peer-review system, which is all easy to use. Visit http:// www.dovepress.com/testimonials.php to read real quotes from published authors. 